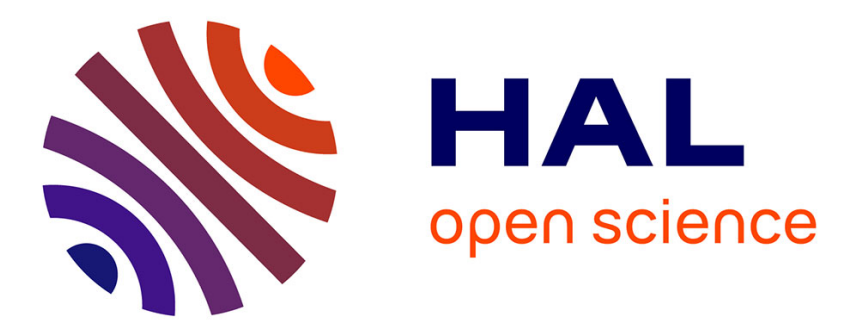

\title{
Mutual Information for Intrapartum fetal Heart Rate Analysis
}

Carlos Granero-Belinchon, Stéphane G. Roux, Nicolas Garnier, Patrice Abry, Muriel Doret

\section{- To cite this version:}

Carlos Granero-Belinchon, Stéphane G. Roux, Nicolas Garnier, Patrice Abry, Muriel Doret. Mutual Information for Intrapartum fetal Heart Rate Analysis. EMBC 2017, Jul 2017, Jeju Island, South Korea. ensl-01637094

\section{HAL Id: ensl-01637094 https://hal-ens-lyon.archives-ouvertes.fr/ensl-01637094}

Submitted on 17 Nov 2017

HAL is a multi-disciplinary open access archive for the deposit and dissemination of scientific research documents, whether they are published or not. The documents may come from teaching and research institutions in France or abroad, or from public or private research centers.
L'archive ouverte pluridisciplinaire HAL, est destinée au dépôt et à la diffusion de documents scientifiques de niveau recherche, publiés ou non, émanant des établissements d'enseignement et de recherche français ou étrangers, des laboratoires publics ou privés. 


\title{
Mutual Information for Intrapartum fetal Heart Rate Analysis
}

\author{
Carlos Granero-Belinchon ${ }^{(1)}$, Stéphane G. Roux ${ }^{(1)}$, Nicolas B. $\operatorname{Garnier}^{(1)}$, Patrice Abry $^{(1)}$, Muriel Doret $^{(2)}$
}

\begin{abstract}
The analysis of the temporal dynamics in intrapartum fetal heart rate (FHR), aiming at early detection of fetal acidosis, constitutes an intricate signal processing task, that continuously receives significant research efforts. Entropy and entropy rates, envisaged as measures of complexity, often computed via popular implementations referred to as Approximate Entropy (ApEn) or Sample Entropy (SampEn), have regularly been reported as significant features for intrapartum FHR analysis. The present contribution aims to show how mutual information enhances characterization of FHR temporal dynamics and improves fetal acidosis detection performance. To that end, mutual information is first connected to ApEn and SampEn both conceptually and with respect to estimation procedure. Second, mutual information, ApEn and SampEn are computed on a large $(\simeq 1000$ subjects) and documented database of FHR data, collected in a French academic hospital. Reported results show that the use of mutual information permits to significantly outperform ApEn and SampEn for acidosis detection, during any stage of labor.
\end{abstract}

\section{INTRODUCTION}

Intrapartum fetal heart rate monitoring. Intrapartum fetal heart rate (FHR) monitoring is a routine procedure in hospitals world-wide, aiming to assess the health status of the baby and to detect as early as possible fetal acidosis, whose occurrence imply rapid intervention to prevent severe consequence for the baby and the mother [1]. In clinical practice, intrapartum FHR analysis essentially relies on the visual inspection of cardiotochograms, guided by a set of rules established by the International federation fo Gynecology and Obstetrics [2]. However, there are on-going research efforts aiming to develop statistical signal processing features permitting to automatically quantify FHR complex temporal dynamics and improve fetal acidos detection.

Related works. Spectrum estimation is amongst the first statistical tool that has been considered for computerized analysis of FHR (cf. e.g., [3], [4]). Aiming to explore temporal dynamics beyond the mere temporal correlations, several variations of nonlinear analysis have been envisaged (cf. e.g., [5], [4], [6]). More recently the concepts of fractal [7], [8] and multifractal [9] have also been showing promising performance in fetal acidosis detection. Complexity measures also constitute classical nonlinear dynamics analysis tools, the most popular being based on information theoretic quantities, based on Shannon Entropy thus permitting to define entropy rates [10], [11], [12], see also [13] for a review. Notably, two practical implementation (or estimation procedure)

\footnotetext{
(1) Univ Lyon, Ens de Lyon, Univ Claude Bernard, CNRS, Laboratoire de Physique, F-69342 Lyon, France firstname. lastnamedens-lyon.fr

(2) Femme-Mère-Enfant Hospital, Bron,, Univ. Lyon I, Lyon, France muriel.doret-dion@chu-lyon.fr
}

of such entropy rates and variations, referred to Approximate Entropy (ApEn) [10], Sample Entropy (SampEn) [14], are massively used in heart rate analysis both for adults and fetuses (cf. e.g., [4], [15]) and are usually considered as state-of-the-art tools in FHR analysis, together with a later extension, Multiscale Entropy (ME), that computes Sample Entropies for several low-pass filtered version of FHR data [16].

Goals, contributions and outline. Revisiting the theoretical definition of entropy rates, the present contribution first shows that entropy rates can be formally split into two components, one of them, referred to as auto-mutual information (MI), conveying information about nonlinear (or higher order) statistics of the temporal dynamics of the data, MI is hence explicitly related to entropy rates and thus to ApEn and SampEn (cf. Section III-A). The estimation procedures of Shannon Entropy and MI on one hand based on k-nearest neighbor (k-NN) algorithms [17], are explicitly compared to those of ApEn and SampEn on other hand, based on Correlation-Integral based algorithms [10], [16], [18] (cf. Section III-B). Further, these different quantities are computed on a large $(\simeq 1000$ subjects $)$ and documented database of FHR data, collected in a French academic hospital, described in Section II. Results reported and discussed in Section IV indicate that MI permits a much more accurate characterization of nonlinear temporal dynamics in FHR time series yielding significantly improved fetal acidosis detection performance, compared to entropy rates, notably compared to their estimation through the ApEn or SampEn implementations. It is also shown that these increased performance are observed independently during both Phase I and Phase II of labor.

\section{DATA}

Database. FHR were routinely monitored and collected at the academic Hospital Femme-Mere-Enfant, Lyon-Bron, France from 2000 to 2010, were a large database of FHR recordings has been set-up and documented with clinical information regarding delivery conditions as well as the health status of the baby cf. [19]. Notably, pH, measured by blood test immediately after delivery is systematically documented and used as ground-truth: When $\mathrm{pH} \leq 7.05$, the newborn is considered has having suffered from acidosis. Cardiotochograms were recorded using scalp electrode by STAN S21 or S31 system, with 12bit resolution, $500 \mathrm{~Hz}$ sampling rate (STAN, Neoventa Medical, Sweden). For the purpose of this study, FHR tracing with too poor quality (missing data, large gaps, short recordings) were excluded. Datasets. For the present study, two large datasets are 
extracted. Dataset I consists of subjects for which delivery took place during Stage I (or after less less than 15min of stage II) and gathers 913 normal $(\mathrm{N}, \mathrm{pH}<7.05))$ and 26 acidotic $(\mathrm{A}, \mathrm{pH} \geq 7.05)$ subjects. Dataset II gathers FHR for delivery that took place during the second stage (Stage II last more than 20min) and gathers 450 normal (N) and 15 acidotic (A) subjects. FHR tracings are analyzed within a single 20-min long window located either at the end of Stage I (for Dataset I ) or Stage II (for Dataset II).

Time Series. From the lists of RR-interarrivals $\Delta_{k}$ in ms available for each subject, regularly sampled FHR time series in Beat-per-Minutes (BpM) are obtained by interpolation of the samples $\left\{\ldots, 36000 / \Delta_{k}, \ldots\right\}$. The sampling frequency has been set to $f_{s}=10 \mathrm{~Hz}$.

\section{INFORMATION THEORETIC QUANTITIES}

\section{A. Entropy, Entropy rate and Mutual Information}

Shannon Entropy. Let $X$ denote a stationary process. Following [20], we define the $m$-dimensional time-embedded process $X_{t}^{(m, \tau)}$ as:

$$
\mathbf{x}_{t}^{(m, \tau)}=(x(t), x(t-\tau), x(t-2 \tau), \ldots, x(t-(m-1) \tau)) .
$$

The Shannon Entropy of the m-embedded version of process $X, H^{(m, \tau)}(X)$, is defined as the Shannon Entropy $H\left(\mathbf{x}_{t}^{(m, \tau)}\right)$ computed from the joint probability density function of the embedded vector $\mathbf{x}_{t}^{(m, \tau)}$ :

$$
\begin{aligned}
H^{(m, \tau)}(X) & =H\left(\mathbf{x}_{t}^{(m, \tau)}\right) \\
& =-\int_{\mathbb{R}^{m}} p\left(\mathbf{x}_{t}^{(m, \tau)}\right) \log p\left(\mathbf{x}_{t}^{(m, \tau)}\right) \mathrm{d} \mathbf{x}_{t}^{(m, \tau)}(.3)
\end{aligned}
$$

The stationarity of $X$ induces that $H^{(m, \tau)}(X)$ does not depend on time $t$. For embedding $m=1$, the Shannon entropy does not depend on the time delay $\tau$ and we note it simply $H$.

Entropy Rates. The entropy rate of order $m, h^{(m, \tau)}(X)$, measures the change in Shannon entropy induced by increasing the embedding dimension:

$$
h^{(m, \tau)}(X)=H^{(m+1, \tau)}(X)-H^{(m, \tau)}(X)
$$

Mutual Information. The general form of mutual information $I^{(m, p, \tau)}(Y, X)$ quantifies the amount of information shared by (stationary) processes $X$ and $Y$, via associated embedded vectors $\mathbf{x}_{t}^{(m, \tau)}$ and $\mathbf{y}_{t^{\prime}}^{(p, \tau)}$ :

$$
\begin{aligned}
& I^{(m, p, \tau)}(Y, X)\left(t^{\prime}-t\right)=I\left(\mathbf{y}_{t^{\prime}}^{(p, \tau)}, \mathbf{x}_{t}^{(m, \tau)}\right) \\
& =\int_{\mathbb{R}^{m+p}} p\left(\mathbf{y}_{t^{\prime}}^{(p, \tau)}, \mathbf{x}_{t}^{(m, \tau)}\right) \\
& \log \left(\frac{p\left(\mathbf{y}_{t^{\prime}}^{(p, \tau)}, \mathbf{x}_{t}^{(m, \tau)}\right)}{p\left(\mathbf{y}_{t^{\prime}}^{(p, \tau)}\right) p\left(\mathbf{x}_{t}^{(m, \tau)}\right)}\right) \mathrm{d} \mathbf{y}_{t^{\prime}}^{(p, \tau)} \mathrm{d} \mathbf{x}_{t}^{(m, \tau)}
\end{aligned}
$$

which, by stationarity depend only on $t^{\prime}-t$.

Temporal dynamics and Mutual Information. To characterize the nonlinear temporal dynamics of process $X$, we propose here the original use of mutual information for $Y=X$ and $t^{\prime}-t=p \tau$ :

$$
I^{(m, p, \tau)}(X)=I\left(\mathbf{x}_{t+p \tau}^{(p, \tau)}, \mathbf{x}_{t}^{(m, \tau)}\right) .
$$

We will refer to this quantity as auto-mutual information of order $(m, p)$. Intuitively, $I^{(m, p, \tau)}(X)$ measures information shared by successive $m$-point and $p$-point dynamics, as the concatenation of $\mathbf{x}_{t+p \tau}^{(p, \tau)}$ and $\mathbf{x}_{t}^{(m, \tau)}$ corresponds exactly to the $(m+p)$-dimensional time-embedded vector $\mathbf{x}_{t}^{(m+p)}$ :

$$
\left(\mathbf{x}_{t+p \tau}^{(p, \tau)}, \mathbf{x}_{t}^{(m, \tau)}\right)=\mathbf{x}_{t}^{(m+p, \tau)} .
$$

Entropy Rates and Auto-Mutual Information. The entropy rate can be rewritten as:

$$
h^{(m, \tau)}(X)=H(X)-I^{(m, 1, \tau)}(X) .
$$

Interestingly, this splits $h^{(m, \tau)}(X)$ into two contributions: $H(X)$ only depends on the one-point statistics of $X$ and is hence a static property. $I^{(m, 1, \tau)}(X)$ gathers all information conveyed by linear and non linear temporal dynamics, irrespective of the variance of $X$ [21].

For illustration, when $X$ is a stationary jointly Gaussian process, hence fully defined by its variance $\sigma^{2}$ and normalized correlation function $c(\tau)$ (such that $c(\tau=0)=1$ ), one has:

$$
\begin{aligned}
& H^{(m, \tau)}(X)=\frac{m}{2} \log \left(2 \pi e \sigma^{2}\right)+\frac{1}{2} \log \left(\left|\Sigma^{(m)}\right|\right) \\
& h^{(m, \tau)}(X)=\frac{1}{2} \log \left(2 \pi e \sigma^{2}\right)-\frac{1}{2} \log \left(\frac{\left|\Sigma^{(m)}\right|}{\left|\Sigma^{(m+1)}\right|}\right) \\
& I^{(m, 1, \tau)}(X)=\quad \frac{1}{2} \log \left(\frac{\left|\Sigma^{(m)}\right|}{\left|\Sigma^{(m+1)}\right|}\right),
\end{aligned}
$$

where $\Sigma^{(m)}$ is the m-dimensional correlation square matrix of the process and $|\Sigma|=\sigma$.

These equations clearly illustrate that $I^{(m, 1, \tau)}(X)$ consists of the temporal dynamics in $h^{(m, \tau)}(X)$, once the contribution of the static (one-point) statistics has been removed.

\section{B. Estimation procedures}

Nearest Neighbors. To estimate the information theoretic quantities defined above, we propose to use Nearest Neighbors procedures, as promoted by [17], [22] for Entropy and [23] for mutual information. In essence, the $k$-nearest neighbor algorithm computes the distance $\hat{\epsilon}$ at which the $k$-th nearest neighbor of the current point $x_{t}^{(m, \tau)}$ is located. In the following, $\hat{H}$ and $\hat{I}^{(m, p, \tau)}$ denote the resulting estimators of Shannon entropy and mutual information, from which $\hat{h}^{(m, \tau)}$ is derived by plugging $\hat{H}$ and $\hat{I}^{(m, p, \tau)}$ into Eq.(7).

Correlation Integrals, Approximate Entropy (ApEn) and Sample Entropy (SampEn). Aiming to compare the proposed quantities and estimation procedures, we recall elementary facts about Approximate Entropy [24] and Sample Entropy [25], [26], classically and massively used to quantify nonlinear complexities in biomedical data. These quantities naturally depend on $\tau$. However, for ease of exposition, the dependency on $\tau$ is herefafter omitted.

Up to a constant, ApEn can be considered an estimate of the entropy rate $h$, while SampEn constitutes an estimate of the order 2 Renyi entropy rate [27]. These estimates 

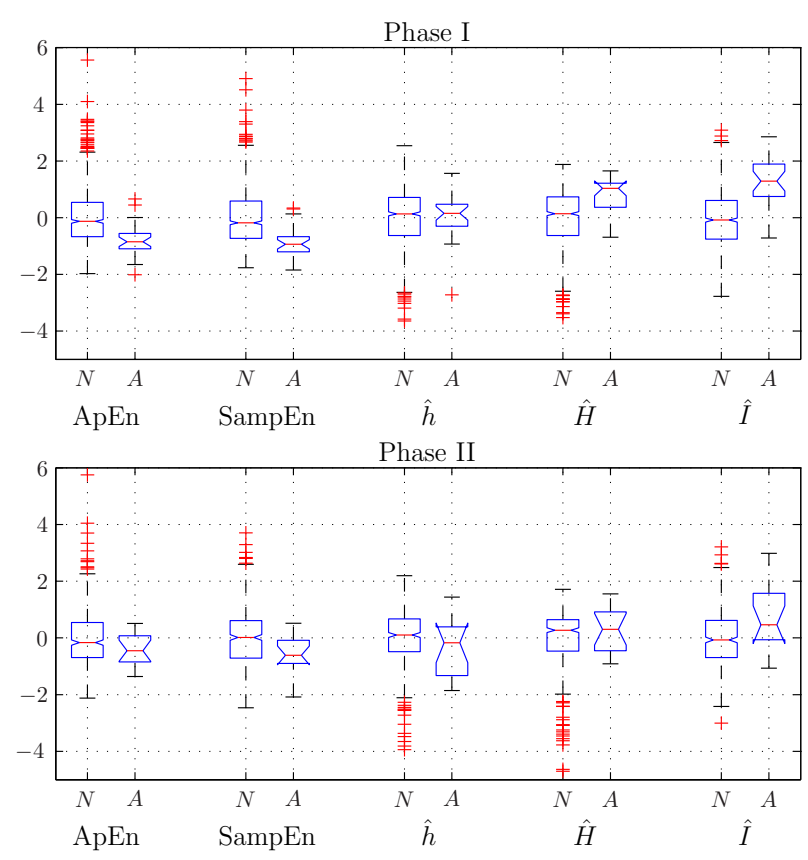

Fig. 1. Box plot based comparisons of the five different (normalized) estimates for Normal (N) and pathological (A for "abnormal"), for Stage I (top) and Stage II (bottom).

are however based on using Correlation Integral procedures, which reverse the perspective compared to Nearest Neighbor algorithm: Correlation Integrals are coarse-graining the data at scale $\epsilon$, which amounts to neglect all details at scales finer than $\epsilon$ and outputs the number $\hat{k}$ of neighbors available at that scale. In practice, SampEn is considered to improve on ApEn as it shows lower sensitivity to parameter tuning and data sample size than ApEn [25], [28].

In the following we computed both quantities using our own Matlab implementation (based on Physio-Net packages). The corresponding estimates are $\operatorname{ApEn}^{(m)}$ and $\operatorname{SampEn}^{(m)}$.

\section{INTRAPARTUM FETAL HEART RATE ANALYSIS}

Parameter setting. For the Nearest neighbor based estimations of $\hat{I}^{(m, p)}$ and $\hat{h}^{(m)}$, We set $k=5, m=2$, $p=1$. For the Integral correlation based estimations of $\operatorname{ApEn}^{(m)}$ and SampEn ${ }^{(m)}$, we used the commonly accepted value, $\epsilon=0.2 \sigma$, with $\sigma$ the standard deviation of $X$, and $m=2$. For all quantities, a single $\tau$ was used. Indeed, it is well documented [9] that BpM FHR times series essentially contain no relevant information above $f_{\max }=2 \mathrm{~Hz}$, which leads to set gap $\tau=5=f_{s} / f_{\max }$, actually corresponding to a delay of 0.5 .

Fetal acidosis detection. Estimations are performed on the last 20 minutes of data before delivery, either in Stage I or Stage II. For each stage independently, the ability of the estimated information theoretic quantities to discriminate fetuses suffering from fetal acidosis (referred to as Abnormal, A) from healthy ones (referred to as Normal, N) is studied. For illustration, Fig. 1 reports the box plots of the normalized (zero-mean, unit-variance) estimates for each

\begin{tabular}{||c||c|c||c|c||}
\hline \multicolumn{1}{|c||}{} & \multicolumn{2}{c||}{ Phase I } & \multicolumn{2}{c||}{ Phase II } \\
\hline \multicolumn{1}{|c||}{} & auc & pval & auc & pval \\
\hline $\mathrm{ApEn}^{2}$ & 0.76 & $4.08 \mathrm{e}-06 *$ & 0.61 & $1.33 \mathrm{e}-01$ \\
\hline SampEn $^{(2)}$ & 0.79 & $5.92 \mathrm{e}-07 *$ & 0.67 & $2.35 \mathrm{e}-02 *$ \\
\hline$\hat{h}^{(2)}$ & 0.50 & $9.75 \mathrm{e}-01$ & 0.39 & $1.36 \mathrm{e}-01$ \\
\hline$\hat{H}$ & 0.76 & $8.36 \mathrm{e}-06 *$ & 0.56 & $4.23 \mathrm{e}-01$ \\
\hline$\hat{I}^{(2,1)}$ & $\mathbf{0 . 8 4}$ & $\mathbf{2 . 0 0 e - 0 9 *}$ & $\mathbf{0 . 6 8}$ & $\mathbf{1 . 6 9 e - 0 2} *$ \\
\hline
\end{tabular}

TABLE I

AUC AND P-VAlue. AREa Under ROC CuRVES (AS SHOWN IN Fig. 2) AND P-VALUE OBTAINED FROM THE WILCOXON RANKSUM TEST, FOR EACH OF THE FIVE DIFFERENT ESTIMATES.

five different estimates, for Stage I and Stage II. Table I quantifies the visual representations in Fig. 1 by reporting, for each estimate, the p-value obtained from applying the classical Wilcoxon ranksum test, testing non-parametrically the null hypothesis that there is no difference between the median of the distribution of the estimates for the normal and abnormal classes. Additionally, Fig. 2 compares ROC (Receiver Operational Characteristics) curves for several estimates, the corresponding AUC (Area Under Curve) are also reported in Table I .

Stage I. For Stage I, Table I and Figs. 1 and 2 indicate a striking result: while $\hat{h}^{(2)}$ does not discriminate classes A and $\mathrm{N}, \hat{H}$ and $\hat{I}^{(2,1)}$ do, very clearly. Interestingly, this shows that during Stage I a mere static effect, essentially the variance of the marginal distribution of FHR times series, permit discrimination. This discrimination is however significantly enhanced when using $\hat{I}^{(2,1)}$. This clearly indicates, that beyond the mere variance, temporal dynamics provide a crucial quantity to look at to achieve efficient detection of fetal acidosis. In addition, merging static and dynamics information into $\hat{h}^{(2)}$ yields a masking effect that degrades the discrimination ability.

While $\hat{h}^{(2)}$ estimated by Nearest Neighbor procedures is not significative, ApEn and SampEn, estimating equivalent quantities by means of Integral Correlation procedures, are able to discriminate the classes A and N. As ApEn and SampEn where shown to be poorer estimates than $\hat{h}^{(2)}$ [27], this surprising result can be interpreted as a secondary benefit of biases in the estimation, that for this application act positively in favor of discrimination. This will be further studied. In all cases, $\hat{I}^{(2,1)}$ significantly outperforms all other quantities both in terms of p-value and AUC, thus clearly emphasizing the benefits of using nonlinear temporal dynamics after removing all static information.

Stage II. The results reported here for Stage II differ significantly from those of Stage I. First, they show that a decrease in performance of all estimated quantities, thus confirming that the well-known fact that the analysis of Stage II is far more intricate than that of Stage I. Second, only two $\left(\hat{I}^{(2,1)}\right.$ and SampEn) out of the five quantities are now able to perform discrimination, thus showing that temporal dynamics in Stage II differ notably for those of Stage I, as recently evidenced quantitatively in [29]. Interestingly the sole one-point statistics $\hat{H}$ is no longer significant in Stage II. The reasons why SampEN appears significant while ApEn 


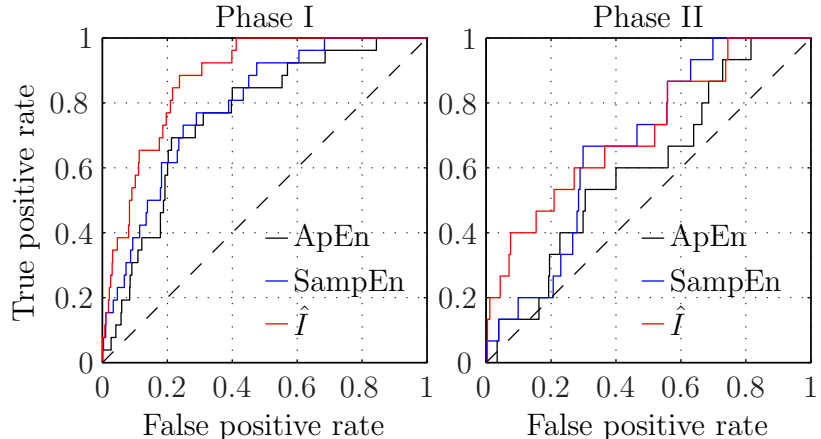

Fig. 2. Compared ROC curves for $\mathrm{SampEn}^{m}, \mathrm{ApEn}^{m}$ and $h^{m}$, for Stage I (left) and Stage II (right)

no longer is remains under investigations. The fact that $\hat{I}^{(2,1)}$ is the quantity that achieves the best performance in terms of p-value and AUC clearly underlines that the analysis of nonlinear temporal dynamics is critical for fetal acidosis detection in Stage II.

\section{CONCLUSIONS AND FUTURE WORKS}

This contribution has promoted the use of mutual information applied to different embedding of BpM FHR times series as a mean to characterize non linear temporal dynamics. It has first proposed to split entropy rates into two terms, one quantifying static properties of the data only versus one associated with nonlinear temporal dynamics. These information theoretic quantities were related to the classical ApEn and SampEn both in terms of interpretations and of estimation issues. Further, applied to two large datasets of FHR data, it has been shown that mutual information constitutes a very promising tool for fetal acidosis detection, both during Stage I and Stage II. The behavior of the several estimated quantities also suggest that temporal dynamics in Stage II differs significantly form those of Stage I, as recently quantified in [29]. These outcome clearly highlight the importance of an accurate analysis of nonlinear dynamics for fetal acidosis detection.

The ability of mutual information to focus on various nonlinear aspect of temporal dynamics by varying the embedding order $m$ and $p$, and by varying $\tau$ are currently being explored, with very promising preliminary results. This is under quantification.

\section{REFERENCES}

[1] E. Chandraharan and S. Arulkumaran, "Prevention of birth asphyxia: responding appropriately to cardiotocograph (CTG) traces.," Best Pract. Res. Clin. Obstet. Gynaecol., vol. 21, no. 4, pp. 609-624, 2007.

[2] FIGO, "Guidelines for the Use of Fetal Monitoring," International Journal of Gynecology \& Obstetrics, vol. 25, pp. 159-167, 1986.

[3] J.O.E.H. Van Laar, et al., "Spectral analysis of fetal heart rate variability for fetal surveillance: Review of the literature," Acta Obstet. Gynecol. Scand., vol. 87, no. 3, pp. 300-306, 2008.

[4] G. Magenes, et al., "Improving the fetal cardiotocographic monitoring by advanced signal processing," Conference proceedings of the IEEE Eng. Med. Biol. Soc., vol. 3, pp. 2295-2298, 2003.

[5] G. Magenes, et al., "Classification of cardiotocographic records by neural networks," Proc. IEEE-INNS-ENNS International Joint Conference on Neural Networks IJCNN, vol. 3, pp. 637-641, 2000.
[6] V. Chudáček, et al., "Scattering transform for intrapartum fetal heart rate variability fractal analysis: a case-control study.," IEEE trans. on Biomed. Eng., vol. 61, pp. 1100-1108, 2014.

[7] D. P. Francis et al., "Physiological basis of fractal complexity properties of heart rate variability in man.," $J$ Physiol, vol. 542, no. Pt 2, pp. 619-629, Jul 2002.

[8] M. Doret, et al., "Fractal analysis and hurst parameter for intrapartum fetal heart rate variability analysis: A versatile alternative to frequency bands and lf/hf ratio.," PloS one, vol. 10, pp. e0136661, 2015.

[9] M. Doret et al., "Multifractal analysis of fetal heart rate variability in fetuses with and without severe acidosis during labor," American Journal of Perinatology, vol. 28, no. 4, pp. 259-266, 2011.

[10] S. M. Pincus and R. R. Viscarello, "Approximate entropy: a regularity measure for fetal heart rate analysis," Obstet Gynecol, vol. 79, no. 2, pp. 249-255, Feb 1992.

[11] S. Pincus, "Approximate entropy (ApEn) as a complexity measure," Chaos, vol. 5, no. 1, pp. 110-117, 1995.

[12] D. E Lake, et al., "Sample entropy analysis of neonatal heart rate variability.," Am J Physiol Regul Integr Comp Physiol, vol. 283, no. 3, pp. R789-R797, Sep 2002.

[13] P. Grünwald and P. Vitányistard, "Kolmogorov complexity and information theory. with an interpretation in terms of questions and answers," Journal of Logic, Language and Information, vol. 12, no. 4, pp. 497-529, 2003.

[14] J. S. Richman and J. R. Moorman, "Physiological time-series analysis using approximate entropy and sample entropy," Am J Physiol Heart Circ Physiol, vol. 278, no. 6, pp. H2039-H2049, Jun 2000.

[15] M. Ferrario, et al., "Comparison of entropy-based regularity estimators: application to the fetal heart rate signal for the identification of fetal distress," IEEE Trans Biomed Eng, vol. 53, no. 1, pp. 119-125, 2006

[16] M. Costa, et al., "Multiscale entropy analysis of complex physiologic time series.," Phys Rev Lett, vol. 89, no. 6, pp. 068102, Aug 2002.

[17] L. F. Kozachenko and N. N. Leonenko, "Sample estimate of the entropy of a random vector," Problems of Information Transmission, vol. 23 , no. 1 , pp. $95-101,1987$

[18] P. Grassberger and I. Procaccia, "Estimation of the kolmogoroventropy from a chaotic signal," Physical Review A, vol. 28, no. 4, pp. 2591-2593, 1983.

[19] M. Doret, et al., "Use of peripartum ST analysis of fetal electrocardiogram without blood sampling: a large prospective cohort study.," Eur J Obstet Gynecol Reprod Biol, vol. 156, no. 1, pp. 35-40, May 2011.

[20] Floris Takens, "Detecting strange attractors in turbulence," Dynamical Systems and Turbulence, vol. 4, pp. 366-381, 1981.

[21] C. Granero-Belinchon, et al., "Scaling of information in turbulence," Epl, vol. 115, no. 5, pp. 58003, Sept. 2016.

[22] L. Kozachenko and N. Leonenko, "Sample estimate of entropy of a random vector," Problems of Information Transmission, vol. 23, pp. 95-100, 1987.

[23] A. Kraskov, et al., "Estimating mutual information," Physical Review $E$, vol. 69, no. 6, pp. 066138, June 2004.

[24] S. M. Pincus, "Approximate entropy as a measure of systemcomplexity," Proc. Natl. Acad. Sci. U.S.A., vol. 88, no. 6, pp. 22972301, Mar. 1991.

[25] J. Richman and R. Moorman, "Time series analysis using approximate entropy and sample entropy.," Biophysical Journal, vol. 78, no. 1, pp. 218A-218A, Jan. 2000.

[26] J. S. Richman, et al., "Sample entropy," Numerical Computer Methods, Pt E, vol. 384, pp. 172-184, 2004.

[27] J. Spilka, et al., "Nearest-neighbor based wavelet entropy rate measures for intrapartum fetal heart rate variability.," Conf. Proc. of the IEEE Eng. Med. Biol. Soc., vol. 2014, pp. 2813-2816, 2014.

[28] D.E. Lake, "Improved entropy rate estimation in physiological data.," Conf. Proc. of the IEEE Eng Med Biol Soc, vol. 2011, pp. 1463-1466, September 2011.

[29] J. Spilka, et al., "Fetal heart rate classification: First vs second stage of labor," in Proc. Int. Workshop Biosignal Interpretation (BSI), Osaka, Japan, 2016. 